\title{
Ecological and genetic factors influencing the transition between host-use strategies in sympatric Heliconius butterflies
}

\author{
R. M. MERRILL* $\dagger^{1}$, R. E. NAISBIT $\ddagger^{1}$, J. MALLET \& \& C. D. JIGGINS* \\ *Department of Zoology, University of Cambridge, Cambridge, UK \\ $\uparrow$ Smithsonian Tropical Research Institute, Balboa, Panama \\ Department of Biology, University of Fribourg, Fribourg, Switzerland \\ §epartment of Organismic and Evolutionary Biology, Harvard University, Cambridge, MA, USA
}

\section{Keywords:}

coexistence:

genetic linkage

host-plant use;

Lepidoptera;

speciation.

\begin{abstract}
Shifts in host-plant use by phytophagous insects have played a central role in their diversification. Evolving host-use strategies will reflect a trade-off between selection pressures. The ecological niche of herbivorous insects is partitioned along several dimensions, and if populations remain in contact, recombination will break down associations between relevant loci. As such, genetic architecture can profoundly affect the coordinated divergence of traits and subsequently the ability to exploit novel habitats. The closely related species Heliconius cydno and H. melpomene differ in mimetic colour pattern, habitat and host-plant use. We investigate the selection pressures and genetic basis underlying host-use differences in these two species. Hostplant surveys reveal that $H$. melpomene specializes on a single species of Passiflora. This is also true for the majority of other Heliconius species in secondary growth forest at our study site, as expected under a model of interspecific competition. In contrast, $H$. cydno, which uses closed-forest habitats where both Heliconius and Passiflora are less common, appears not to be restricted by competition and uses a broad selection of the available Passifora. However, other selection pressures are likely involved, and field experiments reveal that early larval survival of both butterfly species is highest on Passiflora menispermifolia, but most markedly so for $H$. melpomene, the specialist on that host. Finally, we demonstrate an association between host-plant acceptance and colour pattern amongst interspecific hybrids, suggesting that major loci underlying these important ecological traits are physically linked in the genome. Together, our results reveal ecological and genetic associations between shifts in habitat, host use and mimetic colour pattern that have likely facilitated both speciation and coexistence.
\end{abstract}

\section{Introduction}

The evolution of ecological specialization generates biological diversity and can result in speciation. Phytophagous insects are often restricted in their use of host plants (Janzen, 1988; Thompson, 1994), and it has been argued that this is a major evolutionary force promoting their diversification (Ehrlich \& Raven, 1964;

Correspondence: Richard Merrill, Department of Zoology, University of Cambridge, Cambridge CB2 1ST, UK.

Tel.: +44 1223 336644; fax: +44 1223 336676;

e-mail: r.merrill@zoo.cam.ac.uk

${ }^{1}$ These authors contributed equally.
Nosil, 2002). Many factors may promote specialist diets, including the need to deal with plant chemistry (Ehrlich \& Raven, 1964), competition for resources (Karban, 1986), enemy-free space (Atsatt, 1981; Jeffries \& Lawton, 1984) and sexual selection (Quental et al., 2007). Similarly, the transition towards a generalist strategy may be favoured by habitat unpredictability (Strong et al., 1984), nutritional requirements (Bernays et al., 1997) or greater resource availability (Thompson, 1982). As populations diverge, host use will reflect a trade-off between selective pressures imposed by the local environment.

The ecological niche of herbivorous insects is partitioned along several dimensions in addition to 
host-plant use. Consequently, the ability to exploit divergent habitats may rely on further adaptations, including those imposed by thermoregulation (San Martin y Gomez \& Van Dyck, 2012), the local community of predators or parasites (Feder, 1995), the signalling environment (Rodriguez et al., 2004), and camouflage (Nosil \& Crespi, 2006) or mimicry requirements (Mallet \& Barton, 1989; Merrill \& Jiggins, 2009). As such, the exploitation of novel environments typically depends on the coordinated evolution of a suite of traits. Genetic architecture can either impede or promote this process. If diverging populations remain in contact, the transition between specialist and generalist diets (in either direction) may be constrained because recombination will break down the associations between loci for host-plant use and other habitatspecific adaptations. This antagonism between selection and recombination may be overcome by tight physical linkage or pleiotropy (Felsenstein, 1981; Kirkpatrick \& Barton, 2006). In addition, genetic associations can act to transfer the effects of selection on one trait onto others, resulting in the coordinated evolution of traits that together allow populations to exploit new environments.

In Heliconius butterflies, the ability to exploit passion vines (Passifloraceae), which are defended by an array of toxic cyanogenic glycosides, seems to have presented great ecological opportunity (Ehrlich \& Raven, 1964; Turner, 1981). These butterflies are well known for their bright warning patterns, which are both under frequency-dependent selection (Mallet \& Barton, 1989; Merrill et al., 2012) and used during mate recognition (Jiggins et al., 2001b). Convergence in warning pattern due to selection for Müllerian mimicry is common between distantly related Heliconius species; however, there is also great diversity in colour patterns, and within a community, different mimicry rings are loosely segregated by forest type (Smiley, 1978b; Mallet \& Gilbert, 1995; Estrada \& Jiggins, 2002).

The closely related species Heliconius cydno and H. melpomene are sympatric across much of Central and northern South America. Where they co-occur, they differ both in habitat use and in mimetic colour pattern: in Panama, for example, H. cydno chioneus is normally found in closed-forest habitats and mimics the black and white pattern of $H$. sapho, whereas $H$. melpomene rosina occurs in secondary forest and mimics the black, red and yellow pattern of $H$. erato (Estrada $\&$ Jiggins, 2002). This shift in mimetic colour pattern is largely controlled by four autosomal Mendelian loci of major effect, two of which consist of several tightly linked elements (Naisbit et al., 2003). Although hybrids are very rare in nature, and interspecific female hybrids are sterile, low rates of gene flow have been demonstrated (Naisbit et al., 2002; Bull et al., 2006; Kronforst et al., 2006a). Heliconius cydno has a generalist larval diet, although restricted to Passiflora, as does H. melpomene in areas where H. cydno is absent. However, in areas of sympatry, the two species differ in their host use: $H$. melpomene is a specialist, typically using only a single species of Passiflora, whereas H. cydno accepts additional species for oviposition. Thus, in addition to disruptive selection acting on warning pattern and strong assortative mating, the two species are isolated by habitat and host-use differences and female hybrid sterility.

The host-use shift in H. melpomene and H. cydno may be an adaptive response to changes in habitat use, but very little is known about its genetic basis or the selective pressures that cause divergence. Here, we begin to address this gap using field and insectary experiments in Panama. We first show that in areas of secondary growth, Passiflora species are clearly partitioned amongst the resident Heliconius community, as would be expected under a model of interspecific competition: most Heliconius species, including H. melpomene, primarily use just one, or a few, Passiflora species. In contrast, H. cydno, which uses closed-forest habitats where both Heliconius and Passiflora are less common, uses a broad selection of the available Passiflora species. We use field experiments to show that early larval survival of both $H$. melpomene and H. cydno is higher on the native host of H.melpomene. Additional ecological factors must explain the broader host-use strategy of H. cydno. Finally, results from genetic crosses indicate that the transition in host use is influenced, at least in part, by an autosomal locus of major effect in physical linkage with mimetic colour pattern. Together, our results reveal ecological and genetic associations between shifts in habitat, host use and mimetic colour pattern that facilitate speciation and coexistence in these butterflies.

\section{Materials and methods}

\section{Field survey of host use}

Host-plant surveys were conducted between August 1998 and March 2000 on Pipeline Road, Soberanía National Park, Panama (987.40 N, 79842.20 W, elevation $60 \mathrm{~m}$ ). Eggs and larvae collected in the field were reared to the adult stage for species identification, because many Heliconius, and in particular H. cydno and $H$. melpomene, are difficult to distinguish during their immature stages. Most individuals were reared on Passiflora biflora, but where the host plant, oviposition site or larval morphology suggested a host specialist, the larva was reared using the host on which it was found.

\section{Larval survival experiments}

We conducted a field experiment in 1999 to determine the suitability of different Passiflora species as hosts and to explore the selective pressures acting on immature stages of $H$. cydno and $H$. melpomene. Larval survival was tested on wild-growing P. menispermifolia (the host of 
H. melpomene) or one of the other four Passiflora species additionally used by $H$. cydno on Pipeline road (Table 1). We collected eggs from H. cydno and H. melpomene females maintained in captivity and allowed them to hatch in plastic cups before transferring them to wild plants. For each individual plant included in the study, a first instar larva of either $H$. cydno or H. melpomene was placed on the vine, revisited after $48 \mathrm{~h}$ to determine mortality and replaced with a larva of the second species, which itself was revisited after $48 \mathrm{~h}$. Larvae were not caged on the plants. As a result, they were free to move around the plant and onto neighbouring foliage, and this may account for some of the recorded 'mortality'. However, first instar H. cydno and H. melpomene readily accept alternative Passiflora hosts and generally remain on the growing shoots and young leaves of the plant (personal observations), and so this is unlikely to have had a large effect.

We used generalized linear mixed models (GLMMs, implemented using the R package lme4), with binomial response and logit link function, to test for the effect of host plant on larval survival over $48 \mathrm{~h}$. We had two fixed effects each with two levels: host type (P. menispermifolia or additional $H$. cydno host) and caterpillar species (H. cydno or H. melpomene). The fully saturated model was simplified in a stepwise manner using likelihood ratio tests, but always retaining individual passion vine as a random factor to avoid pseudoreplication. Importantly, if the interaction term between host type and caterpillar species was significant, it would suggest that any differences in survival were likely the result of physiological adaptations of the species to their respective hosts. In addition, given the differing expectations for the two butterfly species (for $H$. melpomene, we compare survival on native and non-native hosts, whereas for H. cydno, all hosts are native), we conducted post hoc binomial exact tests to compare survival on the two host types for each species of caterpillar individually.

\section{Host-preference experiment}

To investigate the genetic basis of oviposition preference, we tested $H$. cydno and $H$.melpomene, their Fl hybrids (H. cydno mother $\times H$. melpomene father) and offspring of backcrosses to both species. We established stocks of H. cydno and H. melpomene from Gamboa and the nearby Soberanía National Park, Panama, and maintained these in insectaries in Gamboa between September 2007 and August 2009. Mated females were tested individually in choice cages (approximately $1 \times 2 \times 2 \mathrm{~m}$ ) to determine their oviposition preference between Passiflora menispermifolia, on which H. melpomene specializes in the wild, and P. vitifolia, an additional host used commonly by H. cydno (see Table 1). Females were presented P. menispermifolia and P. vitifolia with fresh shoots for oviposition on alternate days and never experienced the same individual plant twice. Female $H$. cydno and H. melpomene lay a few eggs individually each day throughout their lives, and in our insectary trials, eggs were collected daily. We recorded the numbers of eggs found on P. menispermifolia and P. vitifolia for an even number of days after and including that on which the female started to lay. As Fl females are sterile (Naisbit et al., 2002), backcross hybrids were produced by mating Fl males to $H$. cydno and $H$. melpomene females (BC and BM individuals, respectively).

\section{Testing for associations between host use and colour pattern phenotype}

Differences in wing colour pattern between H. cydno and H. melpomene are controlled by four autosomal

Table 1 Host-plant use by wild Heliconiini. Data represent counts of eggs and larvae collected from different species of Passifora in Gamboa and the nearby Soberańa National Park, Panama. Passiflora species: MEN P. menispermifolia; AMB P. ambigua; NIT P. nitida; VIT P. vitifolia; AUR P. auriculata; COR P. coriacea; BIF P. biflora; TRY P. tryphostemmatoides; FOE P. foetida. Superscript denotes subgenus: ${ }^{G}$ Granadilla;

${ }^{\mathrm{D}}$ Distephana; ${ }^{\mathrm{P}}$ Plectostemma; ${ }^{\mathrm{T}}$ Tryphostemmatoides; ${ }^{\mathrm{Dy}}$ Dysosmia. Eggs and larvae were usually found individually, except for the records from Dione juno (one egg cluster) and from $H$. sara and $H$. doris (two egg clusters each).

\begin{tabular}{|c|c|c|c|c|c|c|c|c|c|}
\hline & $\mathrm{MEN}^{\mathrm{G}}$ & $\mathrm{AMB}^{\mathrm{G}}$ & $N I T^{G}$ & $\mathrm{VIT} T^{\mathrm{D}}$ & $A \cup R^{P}$ & $\mathrm{COR}^{\mathrm{P}}$ & $\mathrm{BIF}^{\mathrm{P}}$ & $T R Y^{\top}$ & $\mathrm{FOE}^{\mathrm{Dy}}$ \\
\hline Heliconius cydno & 14 & 1 & 9 & 15 & 19 & - & - & - & - \\
\hline H. melpomene & 146 & - & - & 1 & 1 & - & - & - & - \\
\hline H. ismenius & - & 11 & - & - & - & - & - & - & - \\
\hline H. hecale & 4 & - & 2 & 126 & 1 & - & - & - & - \\
\hline H. erato & - & - & - & - & 3 & 13 & 12 & - & - \\
\hline H. hecalesia & - & - & - & - & - & - & 2 & 1 & - \\
\hline H. sara & - & - & - & - & 46 & - & - & - & - \\
\hline H. doris & - & 205 & - & - & - & - & - & - & - \\
\hline Eueides & 1 & - & 1 & 11 & - & - & - & - & - \\
\hline Dryas julia & - & - & - & 1 & 33 & 8 & 6 & - & - \\
\hline Philaethria dido & 1 & - & - & 19 & - & - & - & - & - \\
\hline Dione juno & - & - & - & 70 & - & - & - & - & - \\
\hline Agraulis vanillae & - & - & - & - & - & - & - & - & 1 \\
\hline
\end{tabular}


Mendelian loci of major effect, two of which consist of several tightly linked elements (Naisbit et al., 2003). This permits a crude quantitative trait locus (QTL) analysis using segregation of phenotypic traits as genetic markers (Merrill et al., 2011). We scored BC females for colour pattern at the $B, A c$ and $Y b$ loci following Naisbit et al. (2003). At these loci, H. melpomene has the genotype $B B, A c A c$ and $y b y b$ and $H$. cydno is $b b$, $a c a c$ and $Y b Y b$ (see Fig. S1). Amongst BC hybrids, individuals heterozygous at the $B$ locus $(B b)$ have a red forewing band (as observed in H. melpomene), whereas homozygous individuals $(b b)$ lack this. The $A c$ locus also segregates in this cross, resulting in the presence $(a c a c)$ or absence (Acac) of the white hourglass shape observed in the forewing cell of $H$. cydno. At the partially dominant $\mathrm{Yb}$ locus, which controls the expression of the yellow hindwing bar seen in Panamanian races of $H$. melpomene $(y b y b)$, heterozygous individuals $(Y b y b)$ show a shadow of the bar formed by melanic scales with altered reflectance and can be distinguished from homozygous individuals $(Y b Y b)$. Further colour pattern loci also segregate in BC individuals, but these were not included in our analysis as wear on the wings meant they could not be scored with confidence, and in any case, most are tightly linked to either $B$ or $Y b$.

To test for genetic associations between colour pattern loci and host use, we used generalized linear models (GLMs) with beta-binomial response distribution and logit link function, implemented using the $\mathrm{R}$ package aod (Lesnoff \& Lancelot, 2010). We modelled the response vector of the number of eggs laid on P. menispermifolia vs. the number of eggs laid on P. vitifolia. Using a beta-binomial distribution allowed us to account for overdispersion in our data, introduced by the relatively high proportion of females that laid only on P. menispermifolia. To test for additive effects, models were fitted with the three colour pattern loci $(B, A c$ and $Y b)$ as fixed effects each with two levels (homozygous or heterozygous). Significance was then determined by likelihood ratio tests (LRTs) examining the change in deviance following the removal of each variable. GLMs with beta-binomial error distribution were also used to compare the host-plant acceptance of $\mathrm{BC}$ females with that of the Fl and with $H$. cydno females.

\section{Results}

\section{Host use by $H$. cydno and $H$. melpomene}

Our field surveys yielded a total of 784 heliconiine eggs and larvae collected from nine species of Passiflora (Table 1). The only other herbivores found on Passiflora vines were flea beetles (Coleoptera: Chrysomelidae: Alticinae), which were rarely encountered, larvae of Josia (Lepidoptera: Notodontidae: Dioptinae) and larvae of Nymphidium mantus, Theope lycaenina and Juditha molpe (Lepidoptera: Riodinidae). In particular, the results show that $H$. melpomene rosina is a specialist on Passiflora menispermifolia, whereas $H$. cydno chioneus females use a broad range of Passiflora species. In fact, $H$. cydno was the only heliconiine that could be considered oligophagous, with records from five Passiflora species evenly distributed amongst the Granadilla, Distephana and Plectostemma subgenera. In contrast, the other species recorded were all specialists, found predominantly on a single species or subgenus (for instance, $H$. erato was found only on members of the Plectostemma subgenus). Our insectary hostchoice experiments (summarized in Table 2) were in agreement with the field data: $H$. cydno females laid eggs on both Passiflora vitifolia and P. menispermifolia in approximately equal numbers, whereas $H$. melpomene females laid only on $P$. menispermifolia.

\section{Larval survival}

In total, we placed $240 \mathrm{H}$. melpomene and H. cydno larvae on wild Passiflora. Overall mortality was high, with only $20 \%$ of larvae surviving for $48 \mathrm{~h}$. Nevertheless, our field experiments reveal that first instar larvae have lower mortality on P. menispermifolia than on other species of passion vine additionally used by $H$. cydno $(2 \Delta \ln L=4.01$, d.f. $=1, \quad P=0.045$; Fig. 1$)$. Both the interaction between host type and caterpillar species $(2 \Delta \ln L=3.49, \quad$ d.f. $=1, \quad P=0.062) \quad$ and $\quad$ caterpillar

Table 2 Oviposition by captive Heliconius melpomene, H. cydno and their hybrids. 'Generalist' refers to females that laid eggs on both Passiflora menispermifolia and P. vitifolia; 'Specialist' refers to females that laid eggs only on P. menispermifolia; 'Sterile' females laid no eggs (see Merrill et al., 2011).

\begin{tabular}{|c|c|c|c|c|c|c|}
\hline & \multicolumn{3}{|c|}{ Number of females } & \multicolumn{2}{|c|}{ Total eggs laid on } & \multirow{2}{*}{$\begin{array}{l}\text { Mean proportion of eggs } \\
\text { laid on } P \text {. vitifolia ( } \pm \text { SE) }\end{array}$} \\
\hline & 'Generalist' & 'Specialist' & 'Sterile' & P. vitifolia & P. menispermifolia & \\
\hline H. melpomene & 0 & 22 & 0 & 0 & 404 & 0 \\
\hline H. cydno & 13 & 0 & 0 & 169 & 135 & $0.53( \pm 0.07)$ \\
\hline $\mathrm{F}_{1}(\mathrm{H}$. cydno $\times \mathrm{H}$. melpomene $)$ & 4 & 9 & 7 & 31 & 214 & $0.08( \pm 0.04)$ \\
\hline Backcross to $H$. melpomene (BM) & 0 & 16 & 2 & 0 & 399 & 0 \\
\hline Backcross to $H$. cydno (BC) & 15 & 6 & 25 & 107 & 391 & $0.21( \pm 0.04)$ \\
\hline
\end{tabular}




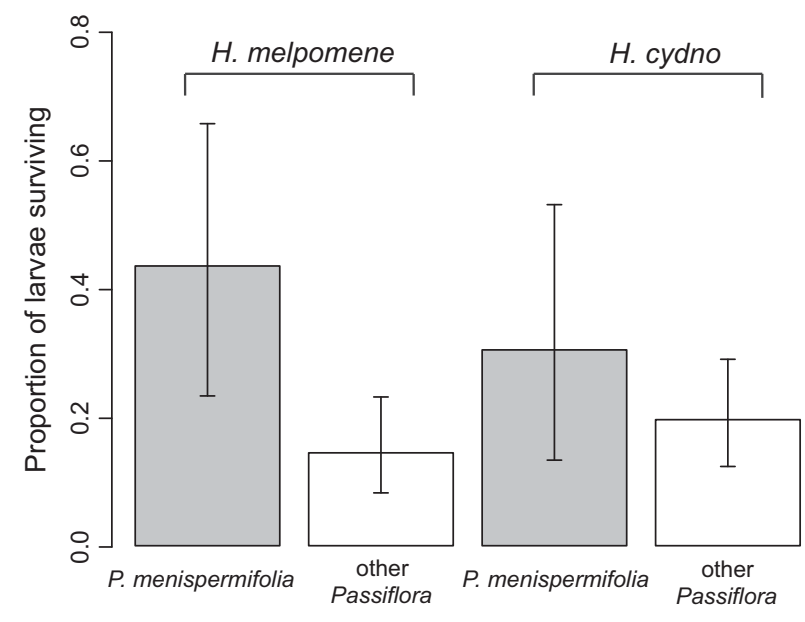

Fig. 1 Survival of first instar larvae on different host plants in the wild. Grey and white bars represent the proportion of larvae surviving $48 \mathrm{~h}$ ( $\pm 95 \%$ confidence intervals) when placed on Passiflora menispermifolia (a host of both butterfly species, $n=23$ ) and on other Passiflora species additionally used by Heliconius cydno $(n=97)$, respectively.

species $(2 \Delta \ln L=0.18$, d.f. $=1, P=0.67)$ were marginally nonsignificant, suggesting a lack of host-specific adaptation between the two butterfly species. However, analysis of the two species of caterpillar separately revealed that larvae of $H$. melpomene were more likely to survive on P. menispermifolia than on the other Passiflora species (10 of 23 larvae (44\%) survived on P. menispermifolia, compared with 14 of $97(14 \%)$ on other Passiflora species; exact binomial test: $P<0.001)$. For $H$. cydno, the difference between the two host types was not statistically significant (seven of 23 larvae (30\%) survived on P. menispermifolia, compared with 19 of 97 $(20 \%)$ on other Passiflora species; exact binomial test: $P=0.191)$.

\section{Genetics of host use and linkage with colour pattern loci}

Of the $13 \mathrm{Fl}$ females that laid eggs in our insectary experiment (from a total of 20), nine used only P. menispermifolia and overall they showed very low acceptance of $P$. vitifolia (Table 2). Consistent with a largely dominant autosomal basis for host-plant acceptance, there was no segregation amongst BM females, all of which laid eggs only on P. menispermifolia (with the exception of two individuals that laid no eggs). Of the $46 \mathrm{BC}$ females tested, 25 laid no eggs (Table 2). Amongst those that did lay eggs, $50-100 \%$ were laid on P. menispermifolia. Overall, the acceptance of $P$. vitifolia was intermediate to that by $\mathrm{Fl}$ and $H$. cydno females: $\mathrm{BC}$ females showed significantly greater acceptance of $P$. vitifolia than $\mathrm{Fl}$ females $(2 \Delta \ln L=4.48$, d.f. $=1$, $P<0.034)$, but significantly less than that by $H$. cydno
$(2 \Delta \ln L=16.3$, d.f. $=1, P<0.005)$. There was segregation amongst the $\mathrm{BC}$ females in their acceptance of $P$. vitifolia associated with the $A c$ locus. Individuals heterozygous at the $A c$ locus that did lay eggs were less likely to oviposit on P. vitifolia (Fig. 2), and removal of $A c$ was the only term to cause a significant reduction in deviance in our GLM $(2 \Delta \ln L=5.82$, d.f. $=1$, $P<0.016)$. Data for individual females are shown in Table S1.

\section{Discussion}

In Central America, H. cydno and H. melpomene use different habitats (Smiley, 1978b; Estrada \& Jiggins, 2002), suggesting that they will rarely be in direct competition for larval hosts. Our host-plant survey, and previous work on Heliconius distribution at our field site and elsewhere (Benson, 1978; Smiley, 1978b; Estrada \& Jiggins, 2002), suggests specialization by $H$. melpomene females is more likely to reflect competition in the second growth community, particularly with H. erato, $H$. ismenius and $H$. hecale, rather than character

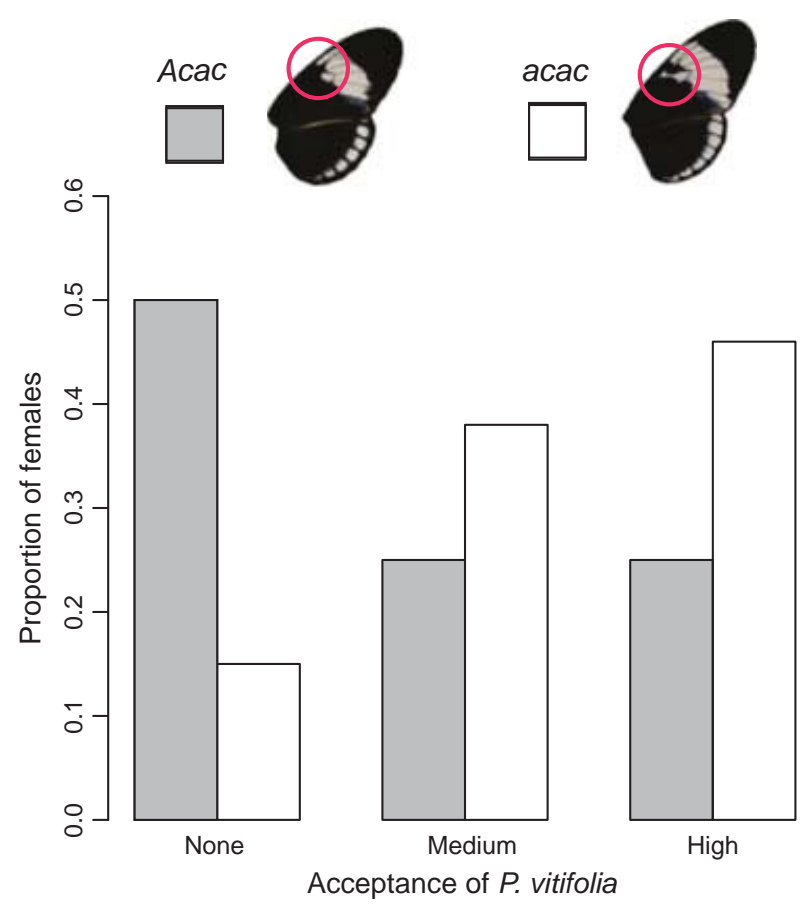

Fig. 2 Host use is associated with the Ac forewing colour pattern locus. The white bars represents female offspring from the backcross to $H$. cydno (BC) that are homozygous at the Ac locus for the $H$. cydno allele and the grey bars represent heterozygous females at the $A c$ locus, having inherited an allele from both H. cydno and H. melpomene. Bars represent the proportion of egglaying females of each genotype that laid no eggs on $P$. vitifolia ('none') had 'medium' acceptance of $P$. vitifolia (0-25\% of eggs) or had 'high' acceptance of $P$. vitifolia (25-50\% eggs). 
displacement with $H$. cydno. In secondary habitats, Passiflora are common but so too are other Heliconius species. Direct competition for larval resources, apparent competition, cannibalism of eggs and larvae by other Heliconius species, and pupal mortality due to misplaced attention from males of pupal-mating species may all play a part in selecting for resource partitioning (Gilbert, 1984, 1991). There is also a second level of partitioning, amongst the Heliconius species, which feed on new growth, and the related genera such as Eueides and Dryas, which tend to feed on older leaves (Benson, 1978). In contrast, in the closed canopy forest used by H. cydno, both Heliconius and fresh Passiflora shoots are rare. In general, Heliconius taxa that use more forested habitats, where suitable Passiflora shoots are sparse, tend be either oligophagous (e.g. H. cydno or H. numata) or specialize on large canopy vines (e.g. H. doris), strategies that may be permitted by the lower diversity of competitors in these habitats. Notably, populations of $H$. melpomene outside the range of $H$. cydno, such as French Guiana, use the forest interior to a greater extent and are more generalist in their larval food plants (Benson et al., 1975).

Our larval survival experiments imply that $P$. menispermifolia is a better host, particularly for H. melpomene. Under laboratory conditions, larval growth rate is similar across these host plants for both butterfly species (Smiley, 1978a), so additional ecological factors, rather than host chemistry, must play a role. One possible explanation is the presence of ants, which are attracted to the extra-floral nectaries of Passiflora and strongly influence early larval survival in Heliconius (Smiley, 1985, 1986). Differential ant attendance at P. quadrangularis and $P$. vitifolia has been suggested as a selective pressure responsible for a preference for the former by H. ismenius in Costa Rica (Smiley, 1986). Although further experimental manipulations are required, reduced attendance by ants on $P$. menispermifolia could thus explain the difference in larval survival (Naisbit, 2002). Although the statistical interaction between Heliconius species and host type was marginally nonsignificant in the analysis of the full data set, post hoc tests for each species separately suggest host-specific adaptation of H. melpomene on P. menispermifolia. A far larger and longer-term experiment would be needed to confirm this, but our data, which already included 240 larvae, are nonetheless intriguing and suggest behavioural or ecological adaptation affecting short-term survival. Further work investigating the fitness tradeoffs associated with different host-use strategies in Heliconius is needed to understand the evolution of shifts in this important ecological trait. Of course, other factors unrelated to larval performance may also influence the host-use strategy adopted. For example, if mating is associated with the host plant (as has been shown in other Heliconius species; Estrada \& Gilbert, 2010), or if larval diet influences pheromonal signals used in mate choice
(Quental et al., 2007), host fidelity might conceivably evolve through a Fisherian runaway process.

\section{Autosomal control of differences in host acceptance between $H$. melpomene and $H$. cydno}

Amongst the Lepidoptera, a disproportionate number of traits distinguishing closely related species are sexlinked (Sperling, 1994; Prowell, 1998). In contrast, our results imply that differences in host use in $\mathrm{H}$. melpomene and $H$. cydno are controlled by autosomal genes. If loci on the Z-chromosome were important, we would have expected host preference to segregate in both backcrosses (Fig. S2), yet all BM females laid only on P. menispermifolia. In addition, specialization on P. menispermifolia was the predominant behaviour in Fl hybrids: of 245 eggs laid by Fl females, only 31 were laid on $P$. vitifolia, suggesting that preference for $P$. menispermifolia is largely a dominant phenotype. However, given the occasional oviposition on P. vitifolia, the possibility remains that preference is a quantitative threshold trait, with a strong preference for $P$. menispermifolia in the Fl, but increasingly cydno-like behaviour with repeated backcrossing to $H$. cydno.

Outside Heliconius, most accounts of the genetic basis of host-plant shifts in Lepidoptera imply a role for genes on the sex chromosomes (Janz, 1998, 2003; Thompson, 1988; Prowell, 1998; Nygren et al., 2006; but see Sheck \& Gould, 1995; Hora et al., 2005). There are several theoretical reasons why sex linkage is expected (reviewed in Qvarnströn \& Bailey, 2009). For example, recessive alleles on the Z-chromosome are exposed to selection in female butterflies (the heterogametic sex in the Lepidoptera) and are therefore expected to evolve more quickly (Charlesworth et al., 1987). In addition, it has been asserted that the reduced rate of recombination of the sex chromosomes will facilitate the build up of 'coadapted gene complexes' and that this might account for the disproportionate number of sex-linked traits in Lepidoptera (e.g. Janz, 2003). Physical linkage will indeed slow the breakdown of associations between taxonspecific traits when gene flow persists (Servedio, 2009); however, recombination is limited to the homogametic sex in Lepidoptera, so in this case, there is no reason to expect reduced recombination rates on the Z-chromosome relative to the autosomes.

Perhaps the lack of sex linkage observed in our study is not unexpected, given that, with the exception of intrinsic hybrid sterility (in the form manifested as the laying of eggs that fail to hatch; Jiggins et al., 200la; Naisbit et al., 2002), no traits distinguishing Heliconius taxa have been shown to be affected by loci on the sex chromosomes. None of the wing patterning loci identified to date are sex-linked (Sheppard et al., 1985; Linares, 1996; Jiggins \& McMillan, 1997; Naisbit et al., 2003); similarly, shifts in mate preference behaviours seem to be largely controlled by autosomal loci 
(Kronforst et al., 2006b; Chamberlain et al., 2009; Merrill et al., 2011). However, linkage between autosomal colour pattern loci and other traits affecting reproductive and ecological isolation has been observed (Kronforst et al., 2006a; Chamberlain et al., 2009; Merrill et al., 2011).

\section{Linkage between host use and other traits affecting reproductive isolation}

Here, we have shown that $\mathrm{BC}$ females that were homozygous for the cydno allele at the $A c$ locus were more likely to lay eggs on both species of Passiflora (i.e. the 'cydno-like' behaviour) compared with their sisters that were heterozygous at this locus (Fig. 2). This implies the existence of a locus, in linkage with $A c$, of considerable effect on host-plant use. Previous work has shown that in the backcross to $H$. melpomene (BM), sterile females typically lay eggs that do not hatch and sterility is associated with the Z-chromosome (Naisbit et al., 2002). By contrast, in the backcross to H. cydno, sterility is usually behavioural, manifested as a failure to lay eggs, and there is no evidence of a large Z-chromosome effect. In analyses of the same backcrosses to H. cydno, we have elsewhere demonstrated that this sterility is associated with the Ac locus (Merrill et al., 2011). Thus, taken together, our results present an example where major loci for three traits affecting reproductive and ecological isolation, involved in host choice, sterility and colour pattern, are located on a single linkage group. Considering that $H$. melpomene and $H$. cydno have 21 chromosome pairs, this is very unlikely to be the result of chance alone [crudely, $P=21 \times(1 / 21)^{3}=$ 0.002]. Other examples of genetic clustering of traits under divergent selection are known. For example, in the pea aphid, QTL controlling host-plant-specific fecundity and host-plant choice comap in four genomic regions (Hawthorne \& Via, 2001). Similarly, amongst Mexican tetra fish, multiple adaptations to a cavedwelling lifestyle cluster in the same genomic regions (Protas et al., 2008).

One intriguing possibility is that the failure to lay eggs could result from the breakdown of oviposition behaviour, partly controlled by the locus identified here. In general, it is understood that loci under divergent selection are ultimately responsible for hybrid sterility (Coyne \& Orr, 2004). Oviposition involves multiple cues and behaviours, including visual inspection and 'drumming' with the forelegs (Benson et al., 1975; Benson, 1978), and it is not inconceivable that the dissociation of coevolved alleles that account for oligophagous or specialist behaviours might cause behavioural sterility. However, although this might explain the colocalization of genes affecting host preference and behavioural sterility, it is of course hard to envision how pleiotropy could explain the additional association with colour pattern.

\section{Implications for speciation and coexistence}

In general, Heliconius diversity is positively correlated with the number of local Passiflora species (Thomas, 1990). Shifts in host use appear to be important in facilitating the final stages of speciation and permitting coexistence. For example, the lack of divergence in host use may prevent $H$. erato and its sister species $H$. himera from entering into sympatry, despite differences in mtDNA, colour pattern and mating preference (Jiggins et al., 1997; McMillan et al., 1997). Similarly, despite strong assortative mating, the hybrid species $H$. heurippa replaces rather than coexists with its putative parental species, H. cydnoltimareta, with which it is, in all but colour pattern, ecologically equivalent (Mavárez et al., 2006; Nadeau et al., 2012). Another parapatric taxon pair within the cydno complex, H. cydno galanthus and H. pachinus, also do not differ in host use. Thus, without further ecological divergence, it seems that evolution is unlikely to proceed past the establishment of parapatric taxa, even though strong premating, and to a lesser extent postmating, isolating barriers may arise. In contrast, $H$. timareta and $H$. melpomene, an unusual case of comimetic sister species with rayed patterns in Colombia and postman patterns in Peru, show striking differences in host use and are sympatric (Giraldo et al., 2008; Mérot et al., 2013). The nonmimetic H. cydno and H. melpomene in Central America provide a second example.

Physical linkage will assist a process by which disruptive selection on colour pattern facilitates divergence at other ecologically relevant loci. Not only is colour pattern under strong disruptive selection for mimicry (Mallet \& Barton, 1989; Merrill et al., 2012), but it is also used as a species-specific mating cue and hybrid females are discriminated against (Jiggins et al., 200lb; Naisbit et al., 2001). Differences in host-plant use may also contribute to premating isolation. Male Heliconius learn the location of Passiflora host plants and patrol them, often mating with teneral females soon after they emerge from pupae on or near their host plant (Estrada $\&$ Gilbert, 2010). It would be interesting to know whether males of specialist species only patrol the 'correct' Passiflora. That major loci underlying changes in colour pattern and host use appear to be physically linked in the genome suggests that the evolutionary histories of these two adaptations are intimately related. A greater understanding of the genetics of ecologically relevant loci influencing multiple niche axes, and how they interact in evolutionary time, will further our knowledge of the speciation process.

\section{Acknowledgments}

We are grateful to Moises Abanto and Timothy Thurman for help in the insectaries. We thank the Smithsonian Tropical Research Institute for support and 
the Ministerio del Ambiente for permission to collect butterflies in Panama. RMM is supported by a Junior Research Fellowship at King's College, Cambridge, and REN was supported by a studentship from the BBSRC and by the Swiss National Science Foundation. We also thank the BBSRC and Leverhulme Trust for funding awarded to CDJ and the BBSRC and NERC for funding awarded to JM.

\section{References}

Atsatt, P.R. 1981. Lycaenid butterflies and ants: selection for enemy-free space. Am. Nat. 118: 638-654.

Benson, W. 1978. Resource partitioning in passion vine butterflies. Evolution 32: 493-518.

Benson, W., Brown, K. \& Gilbert, L. 1975. Coevolution of plants and herbivores: passion flower butterflies. Evolution 29: 659-680.

Bernays, E.A., Angel, J.E. \& Augner, M. 1997. Foraging by a generalist grasshopper: the distance between food resources influences diet mixing and growth rate (Orthoptera: Acrididae). J. Insect Behav. 10: 829-840.

Bull, V., Beltrán, M., Jiggins, C.D., McMillan, W.O., Bermingham, E. \& Mallet, J. 2006. Polyphyly and gene flow between non-sibling Heliconius species. BMC Biol. 4: 11.

Chamberlain, N.L., Hill, R.I., Kapan, D.D., Gilbert, L.E. \& Kronforst, M.R. 2009. Polymorphic butterfly reveals the missing link in ecological speciation. Science 326: 847-850.

Charlesworth, B., Coyne, J.A. \& Barton, N. 1987. The relative roles of evolution of sex chromosomes and autosomes. Am. Nat. 130: 113-146.

Coyne, J.A. \& Orr, H.A. 2004. Speciation. Sinauer, Sunderland, MA, USA.

Ehrlich, P. \& Raven, P. 1964. Butterflies and plants: a study in coevolution. Evolution 18: 586-608.

Estrada, C. \& Gilbert, L.E. 2010. Host plants and immatures as mate searching cues in Heliconius butterflies. Anim. Behav. 80: 231-239.

Estrada, C. \& Jiggins, C.D. 2002. Patterns of pollen feeding and habitat preference among Heliconius species. Ecol. Ent. 27: $448-456$

Feder, J.L. 1995. The effects of parasitoids on sympatic host races of Rhagoletis pomonella (Diptera, Tephritidae). Ecology 76: 801-813.

Felsenstein, J. 1981. Skepticism towards Santa Rosalia, or why are there so few kinds of animals? Evolution 35: 124-138.

Gilbert, L.E. 1984. The biology of butterfly communities. In: The Biology of Butterflies (R.I., Vane-Wright \& P.R., Ackery, eds), pp. 41-54. Academic Press, London, UK.

Gilbert, L.E. 1991. Biodiversity of a Central American Heliconius community: pattern, process, and problems. In: Plant-Animal Interactions: Evolutionary Ecology in Tropical and Temperate Regions (P.W., Price, T.M., Lewinsohn, G.W., Fernandes \& W.W., Benson, eds), pp. 403-427. John Wiley $\&$ Sons, New York, NY, USA.

Giraldo, N., Salazar, C.A., Jiggins, C.D., Bermingham, E. \& Linares, M. 2008. Two sisters in the same dress: Heliconius cryptic species. BMC Evol. Biology 8: 324.

Hawthorne, D. \& Via, S. 2001. Genetic linkage of ecological specialization and reproductive isolation in pea aphids. Nature 412: 904-907.
Hora, K.H., Roessingh, P. \& Menken, S.B.J. 2005. Inheritance and plasticity of adult host acceptance in Yponomeuta species: implications for host shifts in specialist herbivores. Ent. Exp. et App. 115: 271-281.

Janz, N. 1998. Sex-linked inheritance of host-plant specialization in a polyphagous butterfly. Proc. R. Soc. Lond. B 265: $1675-1678$.

Janz, N. 2003. Sex linkage of host plant use in butterflies. In: Butterflies: Ecology and Evolution of Taking Flight (C.L., Boggs, W.B., Watt \& P.R., Ehrlich, eds), pp. 229-239. The University of Chicago Press, Chicago, IL, USA.

Janzen, D.H. 1988. Ecological characterization of a Costa Rican dry forest caterpillar fauna. Biotropica 20: 120-135.

Jeffries, M.J. \& Lawton, J.H. 1984. Enemy free space and the structure of ecological communities. Biol. J. Linn. Soc. 23: 269-286.

Jiggins, C. \& McMillan, W. 1997. The genetic basis of an adaptive radiation: warning colour in two Heliconius species. Proc. R. Soc. Lond. B 264: 1167-1175.

Jiggins, C.D., McMillan, W.O. \& Mallet, J. 1997. Host plant adaptation has not played a role in the recent speciation of Heliconius himera and Heliconius erato (Lepidoptera: Nymphalidae). Ecol. Ent. 22: 361-365.

Jiggins, C.D., Linares, M., Naisbit, R.E., Salazar, C., Yang, Z.H. \& Mallet, J. 2001a. Sex-linked hybrid sterility in a butterfly. Evolution 55: 1631-1638.

Jiggins, C.D., Naisbit, R.E., Coe, R.L. \& Mallet, J. $2001 \mathrm{~b}$. Reproductive isolation caused by colour pattern mimicry. Nature 411: 302-305.

Karban, R. 1986. Interspecific competition between folivorous insects on Erigeron glaucus. Ecology 67: 1063-1072.

Kirkpatrick, M. \& Barton, N. 2006. Chromosome inversions, local adaptation and speciation. Genetics 173: 419-435.

Kronforst, M.R., Young, L., Blume, L. \& Gilbert, L.E. 2006 a. Multilocus analysis of admixture and introgression among Heliconius butterflies. Evolution 60: 1254-1268.

Kronforst, M.R., Young, L.G., Kapan, D.D., McNeely, C., O'Neil, R.J. \& Gilbert, L.E. 2006b. Linkage of butterfly mate preference and wing colour preference at the genomic location wingless. PNAS 103: 6575-6580.

Lesnoff, M. \& Lancelot, R. 2010. Aod: analysis of overdispersed data. R package, version 1.2. http://cran.r-project.org/packagepaod.

Linares, M. 1996. The genetics of the mimetic colouration in the butterfly Heliconius cydno weymeri. J. Hered. 87: 142149.

Mallet, J. \& Barton, N. 1989. Strong natural selection in a warning color hybrid zone. Evolution 43: 421-431.

Mallet, J. \& Gilbert, L.E. 1995. Why are there so many mimicry rings? Correlations between habitat, behaviour and mimicry in Heliconius butterflies. Biol. J. Linn. Soc. 55: 159 180.

Mavárez, J., Salazar, C.A., Bermingham, E., Salcedo, C., Jiggins, C.D. \& Linares, M. 2006. Speciation by hybridization in Heliconius butterflies. Nature 441: 868-871.

McMillan, W., Jiggins, C. \& Mallet, J. 1997. What initiates speciation in passion-vine butterflies? PNAS 94: 8628-8633.

Mérot, C., Mavárez, J., Evin, A., Dasmahapatra, K.K., Mallet, J., Lamas, G. et al. 2013. Genetic differentiation without mimicry shift in a pair of hybridizing Heliconius species (Lepidoptera: Nymphalidae). Biol. J. Linn. Soc. 109: 830-847. 
Merrill, R.M. \& Jiggins, C.D. 2009. Müllerian mimicry: sharing the load reduces the legwork. Curr. Biol. 19: R687R689.

Merrill, R.M., Van Schooten, B., Scott, J.A. \& Jiggins, C.D. 2011. Pervasive genetic associations between traits causing reproductive isolation in Heliconius butterflies. Proc. $R$. Soc Lond. B 278: 511-518.

Merrill, R.M., Wallbank, R.W.R., Bull, V., Salazar, P., Mallet J., Stevens, M. et al. 2012. Disruptive ecological selection on a mating cue. Proc. R. Soc. Lond. B 279: 4907-4913.

Nadeau, N.J., Martin, S.H., Kozak, K.M., Salazar, C., Dasmahapatra, K.K., Davey, J.W et al. 2012. Genome-wide patterns of divergence and gene flow across a butterfly radiation. Mol. Ecol. 22: 814-826.

Naisbit, R.E. 2002. Ecological Divergence and Speciation in Heliconius cydno and H. melpomene. PhD. University College, London.

Naisbit, R.E., Jiggins, C.D. \& Mallet, J. 2001. Disruptive sexual selection against hybrids contributes to speciation between Heliconius cydno and Heliconius melpomene. Proc. R. Soc. Lond. B 268: 1849-1854

Naisbit, R.E., Jiggins, C.D., Linares, M., Salazar, C. \& Mallet, J. 2002. Hybrid sterility, Haldane's rule and speciation in Heliconius cydno and H. melpomene. Genetics 161: 15171526.

Naisbit, R.E., Jiggins, C.D. \& Mallet, J. 2003. Mimicry: developmental genes that contribute to speciation. Evol. Dev. 5: 269-280.

Nosil, P. 2002. Transition rates between specialization and generalization in phytophagous insects. Evolution 56: 17011706

Nosil, P. \& Crespi, B.J. 2006. Experimental evidence that predation promotes divergence in adaptive radiation. Proc Natl. Acad. Sci. 102: 9090-9095.

Nygren, G.H., Nylin, S. \& Stefanescu, C. 2006. Genetics of host plant use and life history in the comma butterfly across Europe: varying modes of inheritance as a potential reproductive barrier. J. Evol. Biol. 19: 1882-1893.

Protas, M., Tabansky, I., Conrad, M., Gross, J.B., Vidal, O., Tabin, C.J. et al. 2008. Multi-trait evolution in a cave fish, Astyanax mexicanus. Evol. Dev. 10: 196-209.

Prowell, D.P. 1998. Sex linkage and speciation in Lepidoptera. In: Endless Forms: Species and Speciation (D.J. Howard, S.H. Berlocher, eds), pp. 309-319. Oxford University Press, New York, NY, USA.

Quental, T.B., Patten, M.M. \& Pierce, N.E. 2007. Host plant specialization driven by sexual selection. Am. Nat. 169: 830836.

Qvarnströn, A. \& Bailey, R.I. 2009. Speciation through evolution of sex-linked genes. Heredity 104: 4-15.

Rodriguez, R.L., Sullivan, L.E. \& Cocroft, R.B. 2004. Vibrational communication and reproductive isolation in the Enchenopa binota species complex of treehoppers (Hemiptera: Membracidae). Evolution 62: 571-578.

San Martin y Gomez, G. \& Van Dyck, H. 2012. Ecotypic differentiation between urban and rural populations of the grasshopper Chorthippus brunneus relative to climate and habitat fragmentation. Oecologia 169: 125-133.
Servedio, M.R. 2009. The role of linkage disequilibrium in the evolution of premating isolation. Heredity 102: 51-56.

Sheck, A.L. \& Gould, F. 1995. Genetic analysis of differences in oviposition preferences of Heliothis virescens and H. subflexa (Lepidoptera: Noctuidae). Envir. Ent. 24: 341-347.

Sheppard, P., Turner, J.R.G., Brown, K., Benson, W. \& Singer, M. 1985. Genetics and the evolution of Muellerian mimicry in Heliconius butterflies. Phil. Trans. R. Soc. B 308: 433-610.

Smiley, J. 1978a. Plant chemistry and the evolution of host specificity: new evidence from Heliconius and Passiflora. Science 201: 745-747.

Smiley, J.T. 1978b. The Host Plant Ecology of Heliconius Butterflies in Northeastern Costa Rica. PhD. University of Texas at Austin, Austin, Texas.

Smiley, J. 1985. Heliconius caterpillar mortality during establishment on plants with and without attending ants. Ecology 66: $845-849$.

Smiley, J. 1986. Ant constancy at Passiflora extrafloral nectaries: effects on caterpillar survival. Ecology 67: 516-521.

Sperling, F.A.H. 1994. Sex-linked genes and species differences in Lepidoptera. Can. Ento. 126: 807-816.

Strong, D.R., Lawton, J.H. \& Southwood, R. 1984. Community Patterns and Mechanisms. Blackwell Scientific, London, UK.

Thomas, C.D. 1990. Fewer species. Nature 347: 702-705.

Thompson, J.N. 1982. Interaction and Coevolution. Wiley, New York, NY, USA.

Thompson, J.N. 1988. Evolutionary genetics of oviposition preference in swallowtail butterflies. Evolution 42: 12231234.

Thompson, J.N. 1994. The Coevolutionary Process. University of Chicago Press, Chicago, IL, USA.

Turner, J.R.G. 1981. Adaptation and evolution in Heliconius: a defence of NeoDarwinism. Ann. Rev. Ecol. Evol. Syst. 12: 99-121.

\section{Supporting information}

Additional Supporting Information may be found in the online version of this article:

Table S1 Data for individual females tested in insectary host preference tests.

Figure S1 The phenotypes of Heliconius melpomene (left), H. cydno (right), and a heterozygote (middle) at the loci studied here: (i) red forewing band $B$ locus, (ii) the anterior part of the white forewing hourglass $A c$ locus, (iii) the yellow hindwing bar $Y b$ locus.

Figure S2 Inheritance of sex-linked ( $\mathrm{W}$ and $\mathrm{Z}$ ) and autosomal (A) alleles in the crosses used to explore host acceptance in Heliconius cydno and H. melpomene.

Data deposited at Dryad: doi:10.5061/dryad.1sr5c 